\title{
Myocardial Blood-Oxygen-Level-Dependent Magnetic Resonance Imaging with Balanced Steady-State Free Precession Imaging Approaches
}

\author{
Rohan Dharmakumar ${ }^{*}$, , Sotirios A. Tsaftaris ${ }^{2}$ and Debiao $\mathrm{Li}^{1}$ \\ ${ }^{I}$ Biomedical Imaging Research Institute, Department of Biomedical Sciences, Cedars-Sinai Medical Center, Los \\ Angeles, CA 90048, USA \\ ${ }^{2}$ Computer Science and Applications, IMT Institutions, Markets, Technologies Institute for Advanced Studies Lucca, \\ Piazza S. Ponziano, 655100 Lucca, Italy
}

\begin{abstract}
The current state of myocardial Blood-Oxygen-Level-Dependent (BOLD) MRI with balanced steady-state free precession (SSFP) approaches is reviewed. Initial studies forming the basis for SSFP-based detection of oxygenation changes beginning with whole blood studies, progressing through controlled studies that consider microcirculatory changes in oxygenation in skeletal muscle and kidney, culminating in basic myocardial studies are outlined. The theoretical basis to observe signal changes and the mechanisms that facilitate such observations are elucidated. Methods to overcome limitations in sensitivity are described.
\end{abstract}

Keywords: BOLD, cardiac, SSFP, blood, oxygen, ischemic heart disease.

\section{INTRODUCTION}

Coronary artery disease is the leading cause of death in the United States [1]. It is estimated that nearly 7 million people are living with coronary artery disease (CAD) in the US and about half a million people die from it each year. The most common form of CAD leads to narrowing of the coronary arteries (stenosis) resulting in reduced blood flow and oxygen supplied to the heart muscle. Accurate early detection of flow deficits may permit interventional revascularization procedures (pharmacological intervention, percutaneous transluminal angioplasty, and/or bypass surgery) to reestablish flow to the hypoperfused regions [2]. The absence of revascularization increases the risk of sudden cardiac death [3].

\section{IMAGING APPROACHES FOR DETECTING CORO- NARY ARTERY DISEASE}

Accurate non-invasive imaging methods for detecting coronary artery disease are necessary to determine which patients should undergo revascularization therapy. The gold standard for detecting coronary artery stenosis is X-ray angiography with iodinated contrast agent which is expensive, invasive, and does not provide information regarding the functional status of the myocardium, which is perhaps more important than morphological information in treating the disease [4-6].

In order to identify $\mathrm{CAD}$ on the basis of functional status of the myocardium, significant research efforts have been devoted to the development of noninvasive methods, but

\footnotetext{
*Address correspondence to this author at the Department of Biomedical Sciences, Cedars-Sinai Medical Center, Biomedical Imaging Research Institute, PACT Bldg - Suite 800; 8700 Beverly Blvd, Los Angeles, 90048, USA; Tel: (310) 423-7641; Fax: (310) 248-8682;

E-mail: rohandkumar@csmc.edu
}

establishment of such methods remains challenging. Current approaches include computed positron emission tomography (PET) and single photon emission computed tomography (SPECT) [7-10]. PET is a promising method for detecting regional myocardial blood flow differences. However, PET studies are limited by low spatial resolution, limited availability, and administration of ionizing radiation. SPECT imaging is the technique most widely used for detecting both metabolic activity and perfusion. However, like PET, SPECT techniques are also limited by low spatial resolution and/or potentially harmful ionizing radiation.

MRI is a noninvasive imaging modality that is gaining increasing acceptance for depiction of vascular anatomy and measurement of cardiac function. MRI has several potential advantages over conventional methods: a) avoids ionizing radiation; b) uses gadolinium chelates as contrast material rather than iodinated contrast media. MR contrast agents are associated with markedly reduced allergic reactions and nephrotoxicity compared with iodinated agents; and c) MRI can provide functional information as well as anatomy in the same setting.

\section{DETECTING CORONARY ARTERY DISEASE WITH MRI}

MRI has been used to directly visualize coronary artery lumen and detect stenoses. Various techniques have been developed and initial clinical studies are promising [11-20]. While significant interest has been placed on identifying stenotic coronary vessels, coronary stenoses do not necessarily result in a negative prognosis $[5,6]$. Thus functional assessment might be a more direct and reliable measure of success for an interventional procedure than methods that identify anatomic lumen changes.

First-pass MRI with gadolinium conjugates has been used for assessing perfusion changes in the setting of coro- 
nary artery disease [21-27]. First-pass methods rely on the detection of changes in myocardial perfusion reserve due to coronary artery disease and thus typically require the use of pharmacological stress agents. This method is evaluated most commonly using rapid imaging techniques with multislice capabilities. While this approach can identify regions of perfusion deficits, the method is limited by inadequate myocardial coverage and sub-optimal temporal and spatial resolution because of the need to capture the first passage of the contrast media at relatively high temporal resolution (1 frame/heartbeat). These limitations can decrease the diagnostic sensitivity and specificity.

\section{MYOCARDIAL BOLD MRI}

An alternate method for identifying perfusion deficits relies on endogenous contrast mechanism mediated by red blood cells. It is known that magnetic susceptibility of red blood cells is determined by the oxygen saturation $\left(\% \mathrm{O}_{2}\right)$ of the hemoglobin. Differential $\% \mathrm{O}_{2}$ of hemoglobin molecules affects the local magnetic field variations in the intra- and the extra-vascular spaces [28-35]. The changes in field inhomogeneities, due to changes in $\% \mathrm{O}_{2}$, are realized as $\mathrm{MR}$ signal changes. This is known as Blood-Oxygen-LevelDependent (BOLD) MRI.

The potential benefits of BOLD MRI for detecting global or regional myocardial ischemia due to $\mathrm{CAD}$ were demonstrated at least a decade ago [36-40]. A number of studies have demonstrated the feasibility of using the MR BOLD effects to assess myocardial blood oxygenation secondary to flow changes in both animals and humans [36-53]. Current BOLD methods rely on deriving oxygen-sensitive contrast with pharmacological stress agents by inducing coronary artery vasodilation, with minimal change in myocardial oxygen demand. Under normal conditions, these stress agents increase baseline coronary venous $\% \mathrm{O}_{2}$ from $20-30 \%$ to $70-80 \%$ [54]. However, the presence of stenosis limits the coronary venous $\% \mathrm{O}_{2}$ from changing markedly during pharmacologic stress, leading to differential venous $\% \mathrm{O}_{2}$ between ischemic and non-ischemic (healthy) myocardium.

The first method used for myocardial BOLD imaging relied on detecting changes in transverse relaxation constant $\mathrm{T}_{2} *$ due to changes in $\% \mathrm{O}_{2}$ using vasodilatory agents [3640]. Although $\mathrm{T}_{2}{ }^{*}$ studies have shown promising results, large magnetic susceptibility artifacts from the lungs [55] have significantly limited the image quality. More robust $\mathrm{T}_{2-}$ prepared methods have provided improved image quality overcoming susceptibility artifacts from the lungs. However, long data acquisition times, cardiac and respiratory motion, and signal modulation during acquisition have been significant obstacles. More recently, balanced steady-state free precession imaging using alternating radio-frequency pulses (SSFP) [53] has been proposed to overcome many of the limitations. SSFP imaging has the following potential advantages over existing MRI methods in the functional assessment of coronary artery disease: (a) cardiac MRI exam without stress-inducing or exogenous MR contrast agents; (b) improved image quality (signal-to-noise ratio and spatial resolution); (c) cardiac phase-resolved BOLD imaging; (d) increased sensitivity to detect regional oxygen deficits; and (e) ability to repeat the exam multiple times within the same imaging.

\section{OXYGEN-SENSITIVE SSFP IMAGING}

Balanced steady-state free precession imaging using alternating radio-frequency pulses (SSFP) has gained widespread recognition for its ability to provide fast scans with high signal-to-noise ratio (SNR). It is the method of choice for cardiac cine imaging at $1.5 \mathrm{~T}$ and is routinely prescribed as part of every clinical CMR (cardiovascular magnetic resonance) exam. The resulting images are analyzed for functional/morphometric changes in the heart, while myocardial signal intensities are typically not evaluated. One of the primary objectives of this review is to demonstrate that myocardial signal intensities from SSFP acquisitions can reflect microcirculatory oxygenation. The sensitivity of myocardial SSFP signal intensities to be modulated by changes in intravascular oxygenation precipitated from initial observation and validation that SSFP signal depends on oxygenation saturation of red blood cells $\left(\% \mathrm{O}_{2}\right)$ in whole blood.

\section{Studies in Whole Blood}

Early observations by Brittain et al., that SSFP imaging enables the discrimination between arteries and veins motivated further investigations to study the sensitivity of SSFP signals to oxygenation changes in whole blood [56]. Systematic whole-blood studies accompanied by theoretical modeling proved that SSFP signals are indeed strongly influenced by oxygen state of whole blood $\left(\% \mathrm{O}_{2}\right)$ [57]. These studies showed that it is the motion of spins through local field inhomogeneities in and around deoxygenated red blood cells that generate SSFP-based image contrast. In particular, it was shown that when spins undergo Brownian motion in blood, they randomly enter and leave the red blood cells while being influenced by the periodic radiofrequency pulses. The extent of their transverse and longitudinal relaxation over the repetition time (TR) in due course determines the bulk steady-state signal. Since $\% \mathrm{O}_{2}$ determines the frequency variations inherent to the medium in which spins undergo diffusion, the $\% \mathrm{O}_{2}$ also affects the steady-state signal. In addition to demonstrating the mechanism associated with oxygen sensitivity, this paper explored the extensive parameter space of SSFP with the intent of optimizing it for robust oxygen-based contrast. Results showed that for a given change in $\% \mathrm{O}_{2}$, when the off-resonance effects are minimized, SSFP signal changes in whole blood are directly dependent on TR, flip angle $(\alpha)$, and field strength $\left(\mathrm{B}_{0}\right)$. As a first application, these basic studies were extended to intravascular 3D peripheral angiographic methods aimed at discriminating arteries and veins based on $\% \mathrm{O}_{2}$ differences $[56,58,59]$. Fig. (1) shows the presence of oxygen-sensitive contrast between an artery and a vein in the leg from one slice of a $3 \mathrm{D}$ acquisition in a human volunteer.

\section{Detection of Microcirculatory Oxygenation Changes with 2D SSFP}

On the basis that whole blood oxygenation changes can be detected with SSFP, rigorous studies were conducted to 


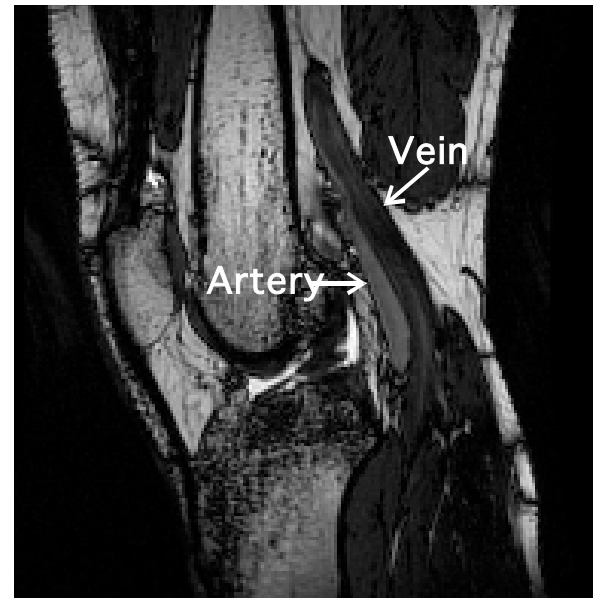

Fig. (1). Sagittal SSFP image of the lower leg of a human volunteer. The image shows a noticeable contrast between the femoral artery and vein which typically differ by about $25 \% \mathrm{O}_{2}$ [femoral vein $\% \mathrm{O}_{2}$ is approximately $70 \%$ and femoral artery $\% \mathrm{O}_{2}$ is approximately 95\%]. Figure adopted from [57].
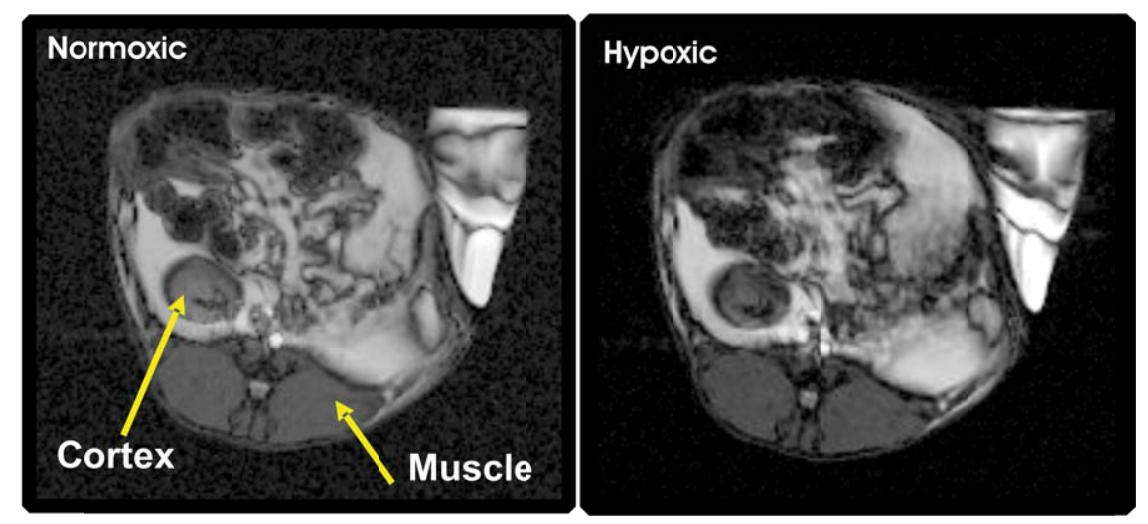

Fig. (2). Typical oxygen-weighted images obtained under normoxic (left) and hypoxic (right) conditions using 2D-balanced SSFP imaging in a healthy rabbit. The arterial blood oxygen saturation at normoxic and hypoxic states in the animal was approximately $88 \%$ and $63 \%$, respectively. Note particularly the signal difference in the kidney under normoxia and hypoxia. Figure adopted from [60].

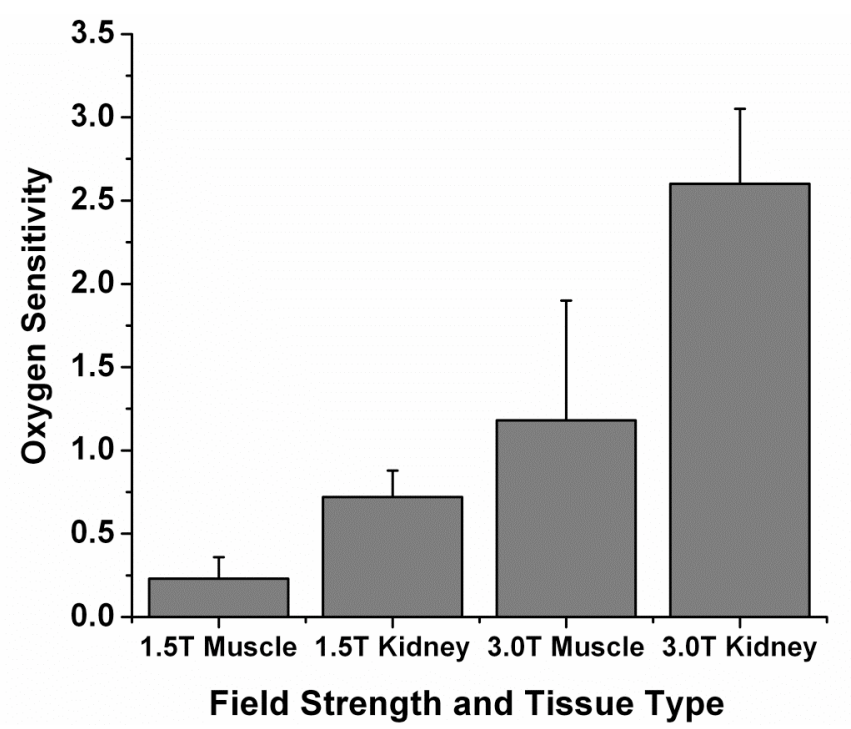

Fig. (3). A bar chart showing experimentally observed mean Oxygen Sensitivity (defined as the relative change in signal difference between normoxic and hypoxic states per $1 \%$ change in arterial blood oxygen saturation), from measured signals under normoxia and systemic hypoxia. The results, reported as means \pm standard deviation, show that field strength and greater blood volume fraction in kidneys directly enhance oxygen sensitivity $(\mathrm{p}<0.001)$. Figure adopted from [60]. 
the greater oxygen sensitivity observed in kidney cortex relative to that of the skeletal muscle is related to the greater blood volume in kidney cortex compared to skeletal muscle (Fig. 2).

\section{Myocardial SSFP-Based BOLD MRI with Provocative Stress at 1.5T in Canines}

The extension of the SSFP approach for evaluating microcirculatory oxygenation changes in the myocardial tissue was first evaluated in a canine model with surgically controllable coronary artery stenosis in the presence of adenosine stress [53]. Animals instrumented with a hydraulic occluder over their left circumflex coronary artery (LCX) underwent CMR studies using 2D SSFP cine imaging.
Compared to conventional cine SSFP imaging that is used for functional assessments, a longer TR was used to ensure BOLD sensitivity. Cine imaging was prescribed to ensure that the magnetization was in steady state. Results showed that regional perfusion deficits due to the presence of LCX stenosis were observable in a cardiac phase dependent manner. The results were compared to the first-pass perfusion technique and correlated against gold-standard microspherebased flow differences. 2D BOLD SSFP method accurately predicted the regional myocardial flow deficit region identified by the first pass technique employing an exogenous contrast media (Fig. 4). Results also showed that it is possible to acquire cardiac phase-resolved BOLD images with 2D SSFP imaging (Fig. 5), albeit a fraction of the diastolic frames were of poor quality due to significant flow artifacts. More-
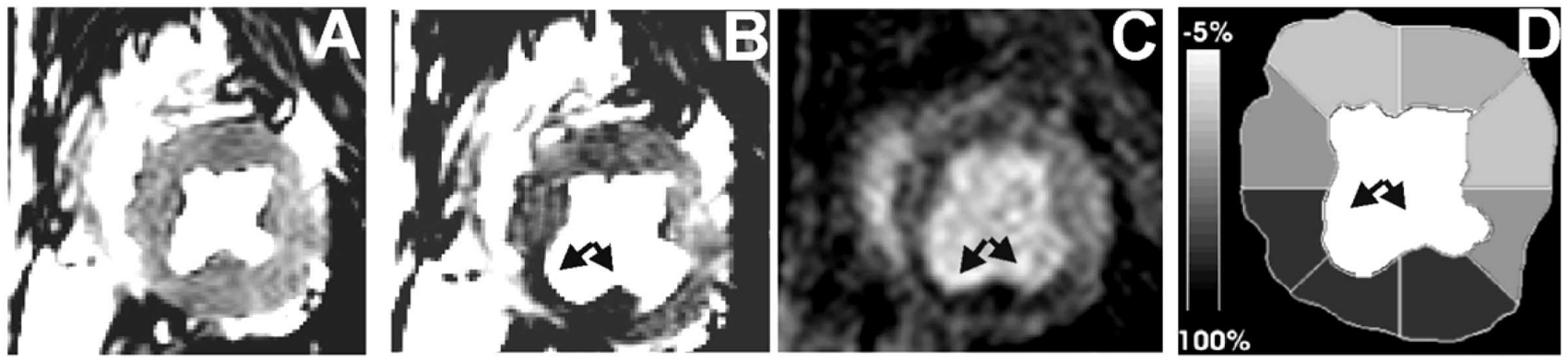

Fig. (4). Typical short axis images showing regional oxygen-sensitive contrast obtained with SSFP-based BOLD technique at pre-stenosis with adenosine (A), severe stenosis $(\mathbf{B})$ at end systole. Image $(\mathbf{C})$ is the associated first-pass perfusion image obtained at the severe stenosis state and image (D) is the spatial map (scale provided by the grey-scale bar) of microsphere-based flow difference between pre-stenosis (with adenosine) and severe stenosis. The arrows subtend the regions where the perfusion deficits are expected to develop due to the stenosis of LCX in dogs. Note the discriminating signal loss in these regions in image B and the close correspondence between the first-pass perfusion and microsphere-based flow map. Figure adopted from [53]
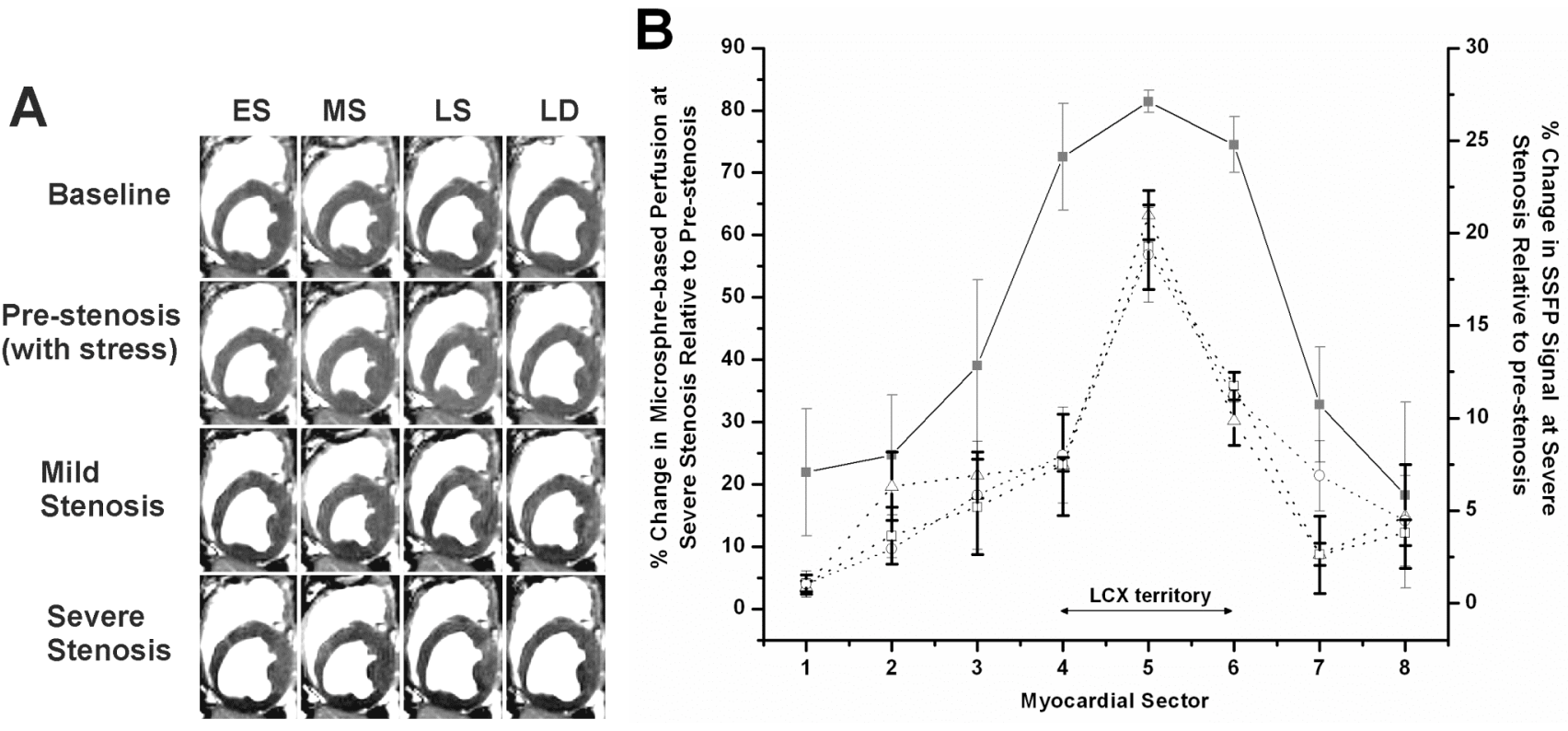

Fig. (5). (A) Typical cardiac phase-resolved BOLD images (early systole (ES), mid systole (MS), late systole (LS), and late diastole (LD)) showing regional myocardial oxygen deficits in the LCX territory during mild and severe stenosis of the left circumflex coronary artery (with adenosine). Note that the extent of signal loss in the LCX territory is related to the extent of LCX stenosis. Baseline and pre-stenosis are also shown at the same cardiac phases for reference. (B) Plot shows the percent change in SSFP-based BOLD contrast at mid-systole (open circles), end-systole (open triangles), and late diastole (open squares) and the associated microsphere-based flow changes (closed squares) observed relative to pre-stenosis over all studies. Note the close correspondence between the MR and microsphere data throughout the myocardium (sectors 1 through 8 ) at all the cardiac phases analyzed. The MR and microsphere measures of relative signal changes are plotted as mean \pm standard error. The dotted black lines (MR) and solid gray lines (microsphere) are provided for visual guidance. Figure adopted from [53]. 
over, although a strong correlation between SSFP signal intensity and true perfusion was established, a direct validation that microcirculatory oxygenation changes mediated the SSFP signal changes was not established by this study.

Earlier studies had accounted for the biophysical mechanisms mediating the detection of oxygenation changes on the basis of SSFP signal intensities. However, these mechanisms do no account for the microvasculature (capillaries) of the heart that contains nearly $90 \%$ of the blood volume. Nearly two decades ago, it was shown that the transverse relaxation changes that facilitate the detection of microcirculatory oxygen changes as BOLD signal changes are strongly dependent on the size of the blood vessel. Although this is well established for gradient-echo and spin-echo imaging methods [34], it remained to be evaluated for SSFP imaging, particularly in the context of myocardial tissue. Recent studies, using Monte-Carlo simulations (MCS), and controlled
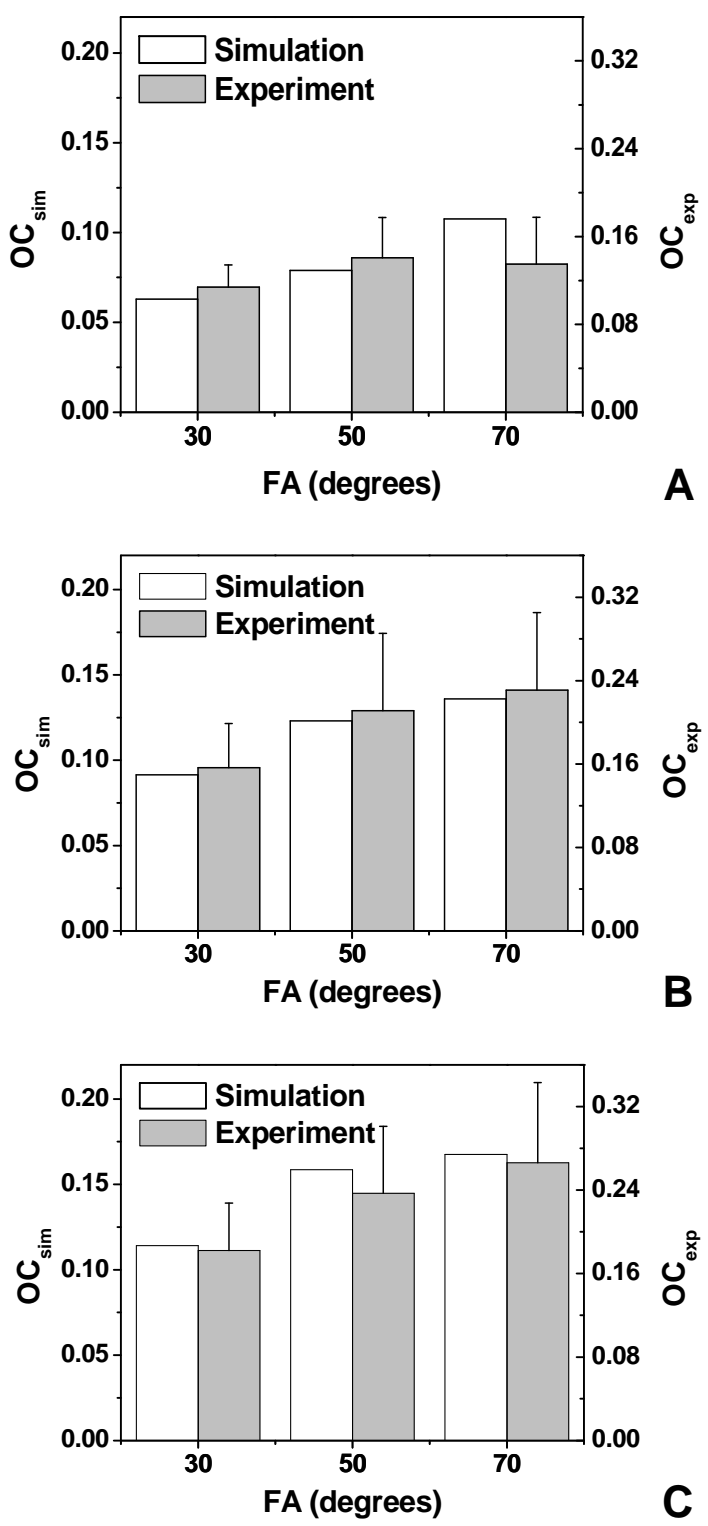

Fig. (6). SSFP-based myocardial BOLD contrast obtained from Monte-Carlo $(\mathrm{MC})$ stimulations $\left(\mathrm{OC}_{\text {sim }}\right)$ and experimental studies $\left(\mathrm{OC}_{\text {exp }}\right)$ with the same parameters $(\mathrm{TR}=3.5 \mathrm{~ms}, 4.7 \mathrm{~ms}$, and $6.0 \mathrm{~ms}$; Flip angle $(\mathrm{FA})=30^{\circ}, 50^{\circ}$, and $\left.70^{\circ}\right)$. Figures adopted from [62]. animal studies have bridged this gap [62]. In the same study, the effect of imaging parameters on SSFP-based myocardial BOLD contrast was also studied using numerical simulations and experimentally validated. Results showed that TR and flip angle play a significant role in determining SSFP-based myocardial BOLD contrast. Both MCS and experimental results showed that increasing the flip angle or TR gave a concomitant increase in SSFP-based myocardial BOLD contrast (Fig. 6). The study also showed that a combination of $\mathrm{TR} / \mathrm{flip}$ angle $=6.0 \mathrm{~ms} / 70^{\circ}$ provided the highest oxygen contrast among all the parameter sets studied $(\mathrm{TR}=3.5,4.7$ and $6.0 \mathrm{~ms} ; \mathrm{FA}=30^{\circ}, 50^{\circ}$ and $70^{\circ}$ ), although image artifacts in some diastolic frames were evident as TR was increased from $3.5 \mathrm{~ms}$ to $6.0 \mathrm{~ms}$.

2D Cine SSFP BOLD imaging does not account for the through-plane motion of heart potentially leading to periodic breakdown of steady state in the tissue being imaged. To address this, a canine study was performed to investigate whether there are any discernable differences in SSFP BOLD contrast between 2D and 3D acquisitions in instrumented canines with LCX occluders. Results from this study showed that oxygen sensitivity between $2 \mathrm{D}$ and $3 \mathrm{D}$ acquisitions were not significantly different, demonstrating that through-plane motion does not significantly alter myocardial SSFP BOLD contrast (Fig. 7). However, to limit the acquisition time to within a single breath hold, the maximum TR that was used in the study was $4.7 \mathrm{~ms}$. To evaluate whether BOLD contrast differences are detectable at longer TRs (e.g. $\mathrm{TR}=6.2 \mathrm{~ms}$ ) between $2 \mathrm{D}$ and $3 \mathrm{D}$ acquisitions, further studies are necessary.

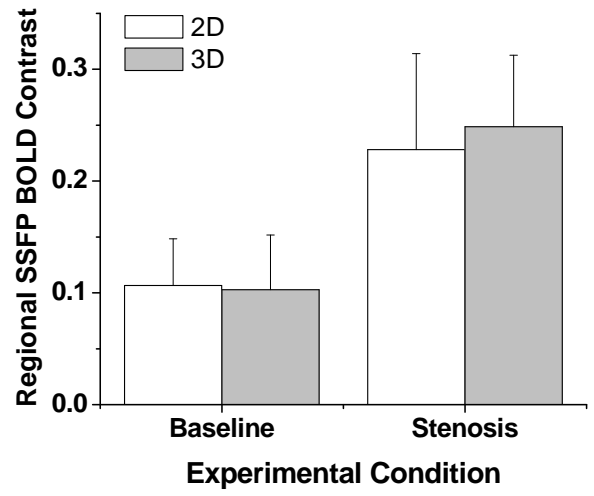

Fig. (7). Regional 2D and 3D SSFP BOLD Contrast between LAD and LCX regions under baseline and stenosis (with adenosine) conditions. While there was no significant Contrast difference between $2 \mathrm{D}$ and $3 \mathrm{D}$ under either condition, a significant Contrast difference was observed between baseline and stenosis conditions $(p<0.01)$ for both $2 \mathrm{D}$ and 3D scans.

\section{SSFP-Based Myocardial BOLD MRI at 3.0T}

Previous theoretical and experimental studies in other tissue types, have clearly demonstrated that SSFP-based oxygen-sensitive contrast is highly dependent on field strength. To evaluate whether such sensitivity patterns can be extended to the myocardial tissue, theoretical simulations and experimental studies using canine models were performed at 1.5T and 3.0T [61]. Theoretical BOLD Contrast, defined as the difference between baseline and hyperemic 

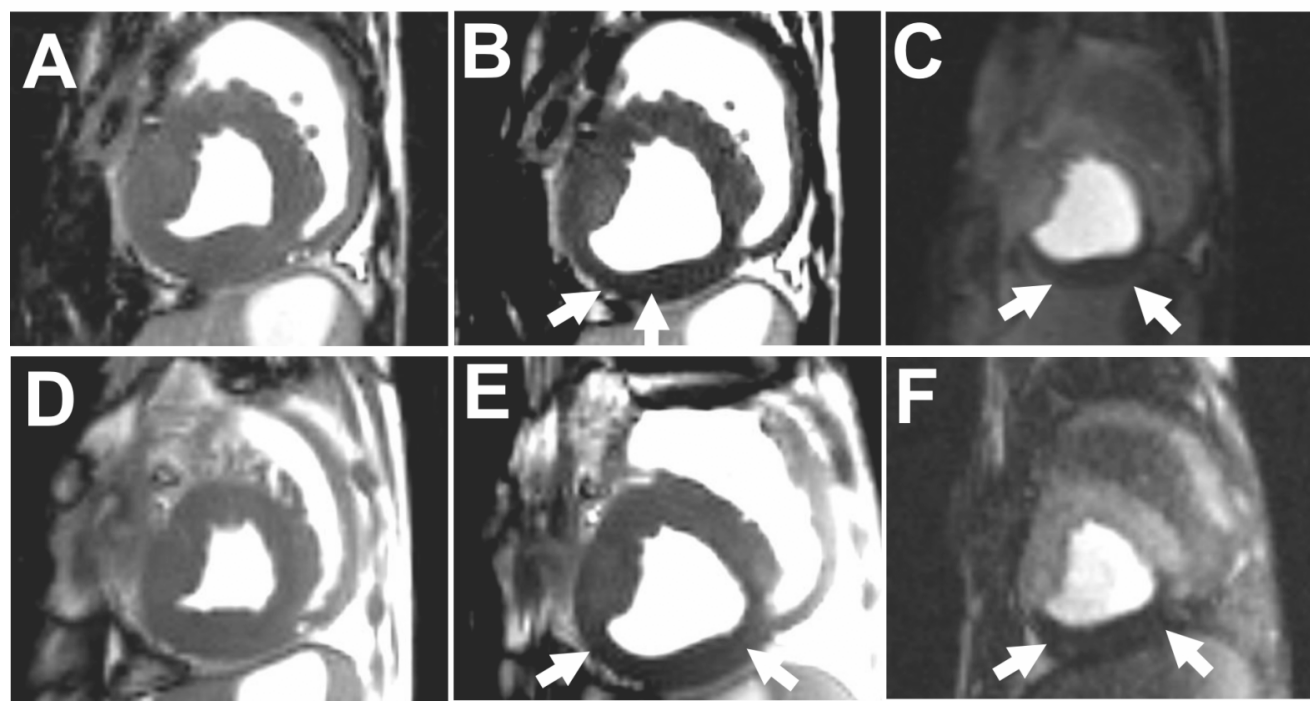

Fig. (8). Typical short axis MR images obtained at 1.5T (top row) and at 3T (bottom row). Images A and D are SSFP images obtained at stress (with no occlusion), images $\mathbf{B}$ and $\mathbf{E}$ are SSFP images at systole under LCX stenosis of similar extent, and images $\mathbf{C}$ and $\mathbf{F}$ are the corresponding first pass images acquired under the same stenosis levels as in B and E, respectively. Note the perfusion deficits and its close correspondence to BOLD images in the LCX territories. Also note the overall improvement in image quality at $3 \mathrm{~T}$ compared to $1.5 \mathrm{~T}$, allowing for a more accurate visualization of oxygen deficit (B and E) in the LCX territory. Figure adopted from [61].

A

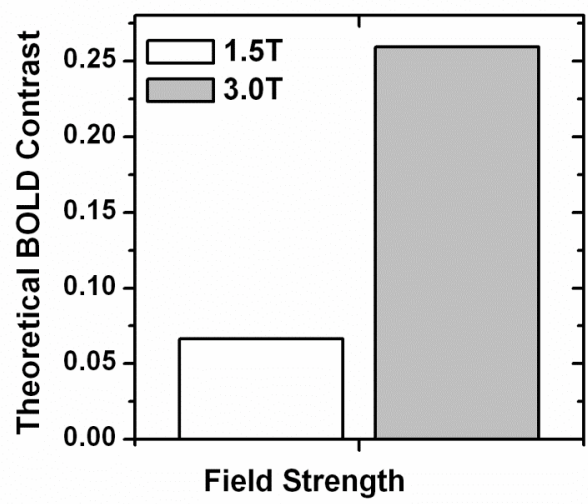

B

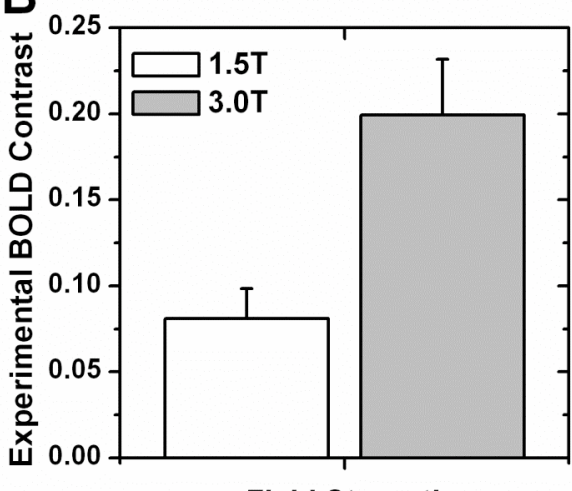

Field Strength

Fig. (9). (A) and (B) Show theoretical and experimental BOLD Contrast at 1.5T and 3.0T. Experimental results confirmed the theoretical prediction that, relative to $1.5 \mathrm{~T}$, significantly greater myocardial BOLD Contrast can be obtained at 3.0T. Figure adopted from [61].

signal intensities normalized by the signal intensity at hyperemic state in the presence of a severe coronary stenosis, was computed. Results showed that compared to $1.5 \mathrm{~T}$, oxygen sensitivity at 3T, should be 3-fold greater. Experimental BOLD Contrast was computed as the difference in signal magnitudes between the LCX territories at baseline and severe stenosis states normalized by the microsphere-based perfusion contrast (computed analogous to BOLD Contrast using fluorescent signals from microsphere). Experimental results (Fig. 8) confirmed the theoretical findings. In particular, a near 3 -fold increase in oxygen sensitivity was observed at 3.0T relative to $1.5 \mathrm{~T}$ (Fig. 9). Experimental findings showed a high correlation between SSFP-signal intensity differences and true perfusion changes ascertained from microsphere flow analysis. However, greater field inhomogeneities at 3.0T due to the susceptibility differences between the heart and lung tissue caused significant difficulties in shimming were notable limitations of the study. Improved shimming strategies that can permit long TR SSFP imaging are necessary to take advantage of the sensitivity gains at 3.0T.

\section{SUMMARY AND OUTLOOK}

Myocardial BOLD MRI using SSFP approaches have the potential to identify regional changes in microcirculatory oxygenation in the presence of provocative (pharmacological) stress. The theoretical basis for signal changes, basic studies in animals have provided a strong basis for further studies. Higher-field imaging studies have shown pathways for improving the sensitivity for assessing BOLD signal changes.

The advancement of SSFP-based myocardial BOLD MRI to the clinical realm will require further studies that demonstrate its utility in patients. Successful patient studies will in turn require acquisition strategies that are fast, generate artifact-free images that can also provide quantitative information relating to the disease state with sufficient sensitivity 
to identify clinically significant conditions, and validated approaches that simplify image analysis.

\section{CONFLICT OF INTEREST}

None declared.

\section{ACKNOWLEDGEMENT}

None declared.

\section{REFERENCES}

[1] Slater J, Rill V. Coronary artery disease: new insights into the pathophysiology, prevalence, and early detection of a monster menace. Semin Ultrasound CT MR 2004; 25: 113-21.

[2] Pagley PR, Beller GA, Watson DD, Gimple LW, Ragosta M. Improved outcome after coronary bypass surgery in patients with ischemic cardiomyopathy and residual myocardial viability. Circulation 1997; 96: 793-800.

[3] Mickleborough LL, Maruyama H, Takagi Y, Mohamed S, Sun Z, Ebisuzaki L. Results of revascularization in patients with severe left ventricular dysfunction. Circulation 1995; 92: I I73-9.

[4] Rackley CE, Russell RO Jr. Left ventricular function in acute and chronic coronary artery disease. Annu Rev Med 1975; 26: 105-20.

[5] Sethna DH, Moffitt EA. An appreciation of the coronary circulation. Anesth Analg 1986; 65: 294-305.

[6] Tayebjee MH, Lip GY, MacFadyen RJ. Collateralization and the response to obstruction of epicardial coronary arteries. QJM 2004; 97: 259-72.

[7] Thornhill RE, Prato FS, Wisenberg G. The assessment of myocardial viability: a review of current diagnostic imaging approaches. J Cardiovasc Magn Reson 2002; 4: 381-410.

[8] Vanoverschelde JL, Gerber B, Pasquet A, Melin JA. Nuclear and echocardiographic imaging for prediction of reversible left ventricular ischemic dysfunction after coronary revascularization: current status and future directions. J Cardiovasc Pharmacol 1996; 28 (Suppl 1): S27-36.

[9] Clark AN, Beller GA. The present role of nuclear cardiology in clinical practice. Q J Nucl Med Mol Imaging 2005; 49: 43-58.

[10] Wei K, Tong KL, Belcik T, et al. Detection of coronary stenoses at rest with myocardial contrast echocardiography. Circulation 2005; 112: $1154-60$

[11] Meyer CH, Hu BS, Nishimura DG, Macovski A. Fast spiral coronary artery imaging. Magn Reson Med 1992; 28: 202-13.

[12] Manning WJ, Li W, Edelman RR. A preliminary report comparing magnetic resonance coronary angiography with conventional angiography. N Engl J Med 1993; 328: 828-32.

[13] Wang Y, Christy PS, Korosec FR, et al. Coronary MRI with a respiratory feedback monitor: the 2D imaging case. Magn Reson Med 1995; 33: 116-21.

[14] Li D, Kaushikkar S, Haacke EM, et al. Coronary arteries: threedimensional MR imaging with retrospective respiratory gating. Radiology 1996; 201: 857-63.

[15] Wang Y, Rossman PJ, Grimm RC, Riederer SJ, Ehman RL. Navigator-echo-based real-time respiratory gating and triggering for reduction of respiration effects in three-dimensional coronary MR angiography. Radiology 1996; 198: 55-60.

[16] Woodard PK, Li D, Haacke EM, et al. Detection of coronary stenoses on source and projection images using three-dimensional MR angiography with retrospective respiratory gating: preliminary experience. AJR Am J Roentgenol 1998; 170: 883-8.

[17] Oshinski JN, Hofland L, Dixon WT, Pettigrew RI. Magnetic resonance coronary angiography using navigator echo gated realtime slice following. Int J Card Imaging 1998; 14: 191-9.

[18] Stuber M, Botnar RM, Danias PG, Kissinger KV, Manning WJ. Submillimeter three-dimensional coronary MR angiography with real-time navigator correction: comparison of navigator locations. Radiology 1999; 212: 579-87.

[19] Deshpande VS, Shea SM, Laub G, Simonetti OP, Finn JP, Li D. 3D magnetization-prepared true-FISP: a new technique for imaging coronary arteries. Magn Reson Med 2001; 46: 494-502.
[20] Kim WY, Danias PG, Stuber M, et al. Coronary magnetic resonance angiography for the detection of coronary stenoses. N Engl J Med 2001; 345: 1863-9.

[21] Wilke N, Simm C, Zhang J, et al. Contrast-enhanced first pass myocardial perfusion imaging: correlation between myocardial blood flow in dogs at rest and during hyperemia. Magn Reson Med 1993; 29: 485-97.

[22] Wilke N, Jerosch-Herold M, Wang Y, et al. Myocardial perfusion reserve: assessment with multisection, quantitative, first-pass MR imaging. Radiology 1997; 204: 373-84.

[23] Wilke NM, Jerosch-Herold M, Zenovich A, Stillman AE. Magnetic resonance first-pass myocardial perfusion imaging: clinical validation and future applications. J Magn Reson Imaging 1999; 10: 676-85

[24] Wyttenbach R, Saeed M, Wendland MF, et al. Detection of acute myocardial ischemia using first-pass dynamics of MnDPDP on inversion recovery echoplanar imaging. J Magn Reson Imaging 1999; 9: 209-14.

[25] Arai AE. Magnetic resonance first-pass myocardial perfusion imaging. Top Magn Reson Imaging 2000; 11: 383-98.

[26] Schreiber WG, Schmitt M, Kalden P, Mohrs OK, Kreitner KF, Thelen M. Dynamic contrast-enhanced myocardial perfusion imaging using saturation-prepared TrueFISP. J Magn Reson Imaging 2002; 16: 641-52.

[27] Jerosch-Herold M, Seethamraju RT, Swingen CM, Wilke NM, Stillman AE. Analysis of myocardial perfusion MRI. J Magn Reson Imaging 2004; 19: 758-70.

[28] Thulborn KR, Waterton JC, Matthews PM, Radda GK. Oxygenation dependence of the transverse relaxation time of water protons in whole blood at high field. Biochim Biophys Acta 1982; 714: 265-70.

[29] Wright GA, Hu BS, Macovski A. 1991 I.I. Rabi Award. Estimating oxygen saturation of blood in vivo with MR imaging at $1.5 \mathrm{~T}$. J Magn Reson Imaging 1991; 1: 275-83.

[30] Meyer ME, Yu O, Eclancher B, Grucker D, Chambron J. NMR relaxation rates and blood oxygenation level. Magn Reson Med 1995; 34: 234-41.

[31] Li D, Wang Y, Waight DJ. Blood oxygen saturation assessment in vivo using T2* estimation. Magn Reson Med 1998; 39: 685-90.

[32] Ogawa S, Lee TM, Kay AR, Tank DW. Brain magnetic resonance imaging with contrast dependent on blood oxygenation. Proc Natl Acad Sci USA 1990; 87: 9868-72.

[33] Kennan RP, Zhong J, Gore JC. Intravascular susceptibility contrast mechanisms in tissues. Magn Reson Med 1994; 31: 9-21.

[34] Boxerman JL, Bandettini PA, Kwong KK, et al. The intravascular contribution to fMRI signal change: Monte Carlo modeling and diffusion-weighted studies in vivo. Magn Reson Med 1995; 34: 4 10.

[35] Zhong J, Kennan RP, Fulbright RK, Gore JC. Quantification of intravascular and extravascular contributions to BOLD effects induced by alteration in oxygenation or intravascular contrast agents. Magn Reson Med 1998; 40: 526-36.

[36] Atalay MK, Forder JR, Chacko VP, Kawamoto S, Zerhouni EA. Oxygenation in the rabbit myocardium: assessment with susceptibility-dependent MR imaging. Radiology 1993; 189: 75964

[37] Balaban RS, Taylor JF, Turner R. Effect of cardiac flow on gradient recalled echo images of the canine heart. NMR Biomed 1994; 7 : 89-95.

[38] Atalay MK, Reeder SB, Zerhouni EA, Forder JR. Blood oxygenation dependence of $\mathrm{T} 1$ and $\mathrm{T} 2$ in the isolated, perfused rabbit heart at 4.7T. Magn Reson Med 1995; 34: 623-7.

[39] Li D, Dhawale P, Rubin PJ, Haacke EM, Gropler RJ. Myocardial signal response to dipyridamole and dobutamine: demonstration of the BOLD effect using a double-echo gradient-echo sequence. Magn Reson Med 1996; 36: 16-20.

[40] Niemi P, Poncelet BP, Kwong KK, et al. Myocardial intensity changes associated with flow stimulation in blood oxygenation sensitive magnetic resonance imaging. Magn Reson Med 1996; 36: 78-82.

[41] Li D, Oellerich WF, Beck G, Gropler RJ. Assessment of myocardial response to pharmacologic interventions using an improved MR imaging technique to estimate T2 values. AJR Am J Roentgenol 1999; 172: 141-5

[42] Wacker CM, Bock M, Hartlep AW, et al. Changes in myocardia oxygenation and perfusion under pharmacological stress with 
dipyridamole: assessment using $\mathrm{T} 2 *$ and $\mathrm{T} 1$ measurements. Magn Reson Med 1999; 41: 686-95.

[43] Wacker CM, Bock M, Hartlep AW, et al. BOLD-MRI in ten patients with coronary artery disease: evidence for imaging of capillary recruitment in myocardium supplied by the stenotic artery. Magma 1999; 8: 48-54.

[44] Beache GM, Herzka DA, Boxerman JL, et al. Attenuated myocardial vasodilator response in patients with hypertensive hypertrophy revealed by oxygenation-dependent magnetic resonance imaging. Circulation 2001; 104: 1214-7.

[45] Wright KB, Klocke FJ, Deshpande VS, et al. Assessment of regional differences in myocardial blood flow using T2-weighted 3D BOLD imaging. Magn Reson Med 2001; 46: 573-8.

[46] Foltz WD, Huang H, Fort S, Wright GA. Vasodilator response assessment in porcine myocardium with magnetic resonance relaxometry. Circulation 2002; 106: 2714-9.

[47] Friedrich MG, Niendorf T, Schulz-Menger J, Gross CM, Dietz R. Blood oxygen level-dependent magnetic resonance imaging in patients with stress-induced angina. Circulation 2003; 108: 221923.

[48] Wacker CM, Hartlep AW, Pfleger S, Schad LR, Ertl G, Bauer WR. Susceptibility-sensitive magnetic resonance imaging detects human myocardium supplied by a stenotic coronary artery without a contrast agent. J Am Coll Cardiol 2003; 41: 834-40.

[49] Fieno DS, Shea SM, Li Y, Harris KR, Finn JP, Li D. Myocardial perfusion imaging based on the blood oxygen level-dependent effect using T2-prepared steady-state free-precession magnetic resonance imaging. Circulation 2004; 110: 1284-90.

[50] Zheng J, Wang J, Nolte M, Li D, Gropler RJ, Woodard PK. Dynamic estimation of the myocardial oxygen extraction ratio during dipyridamole stress by MRI: a preliminary study in canines. Magn Reson Med 2004; 51: 718-26.

[51] Zheng J, Wang J, Rowold FE, Gropler RJ, Woodard PK. Relationship of apparent myocardial T2 and oxygenation: towards quantification of myocardial oxygen extraction fraction. J Magn Reson Imaging 2004; 20: 233-41.

[52] Shea SM, Fieno DS, Schirf BE, et al. T2-prepared steady-state free precession blood oxygen level-dependent MR imaging of myocardial perfusion in a dog stenosis model. Radiology 2005; 236: 503-9.

[53] Dharmakumar R, Arumana JM, Larson AC, Chung Y, Wright GA, Li D. Cardiac phase-resolved blood oxygen-sensitive steady-state free precession MRI for evaluating the functional significance of coronary artery stenosis. Invest Radiol 2007; 42: 180-8.

[54] Li D, Oellerich WF, Gropler RJ. Magnetic resonance assessment of myocardial oxygenation. In: Manning W, Pennell D, Eds. Cardiovascular Magnetic Resonance. Philadelphia, PA: Churchill Livingstone 2001; 447-54.

[55] Reeder SB, Faranesh AZ, Boxerman JL, McVeigh ER. In vivo measurement of $\mathrm{T}^{*}$ and field inhomogeneity maps in the human heart at 1.5 T. Magn Reson Med 1998; 39: 988-98.

[56] Brittain JH, Shimakawa A, Johnson JW, et al. SSFP Non-ContrastEnhanced MR Angiography. In: MRA Workshop. Beijing, China $2005 ;$ p. 189.

[57] Dharmakumar R, Hong J, Brittain JH, Plewes DB, Wright GA. Oxygen-sensitive contrast in blood for steady-state free precession imaging. Magn Reson Med 2005; 53: 574-83.

[58] Brittain JH, Reeder SB, Shimakawa A, et al. Non-ContrastEnhanced Angiography at 3T using SSFP and "Dixon" Fat-Water Separation. Proceedings of the $12^{\text {th }}$ Annual Meeting of ISMRM; Kyoto 2004; p. 12.

[59] Brittain JH, Shimakawa A, Johnson JW, et al. SSFP Non-ContrastEnhanced MR Angiography at 3.0T: Improved Arterial-Venous Contrast with Increased TR. In: ISMRM 2005.

[60] Dharmakumar R, Qi X, Hong J, Wright GA. Detecting microcirculatory changes in blood oxygen state with steady-state free precession imaging. Magn Reson Med 2006; 55: 1372-80.

[61] Dharmakumar R, Arumana J, Tang R, Harris K, Zhang Z, Li D. Assessment of regional myocardial oxygenation changes in the presence of coronary artery stenosis with balanced SSFP imaging at 3.0T: Theory and experimental evaluation in canines. J Magn Reson Imaging 2008; 27: 1037-45.

[62] Zhou, X, Tang R, Klein R, Li D, Dharmakumar R. Parametric dependence of myocardial blood-oxygen-level-dependent steadystate free precession imaging at $1.5 \mathrm{~T}$ : theory and experiments. Magn Reson Med 2010; 63(2): 484-93.

Received: December 29, 2010

Revised: February 09, 2012

Accepted: February 13, 2012

(C) Dharmakumar et al.; Licensee Bentham Open.

This is an open access article licensed under the terms of the Creative Commons Attribution Non-Commercial License (http:/creativecommons.org/ licenses/by-nc/3.0/), which permits unrestricted, non-commercial use, distribution and reproduction in any medium, provided the work is properly cited. 